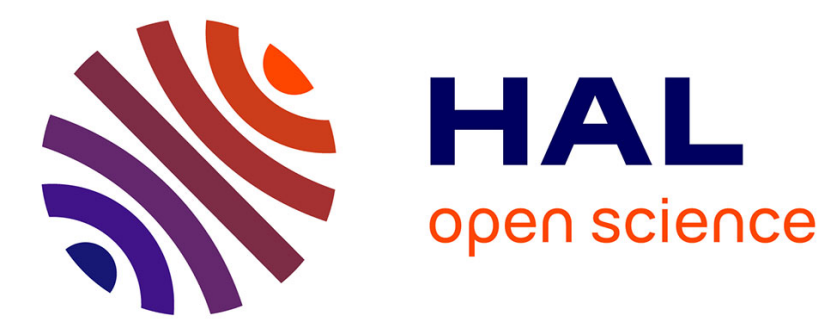

\title{
A la recherche de la centralité perdue
}

\author{
Lise Bourdeau-Lepage, J.M. Huriot, J. Perreur
}

\section{To cite this version:}

Lise Bourdeau-Lepage, J.M. Huriot, J. Perreur. A la recherche de la centralité perdue. Revue d'économie régionale et urbaine, 2009, 3, pp.549 -572. hal-00453859

\section{HAL Id: hal-00453859 \\ https://hal.science/hal-00453859}

Submitted on 5 Feb 2010

HAL is a multi-disciplinary open access archive for the deposit and dissemination of scientific research documents, whether they are published or not. The documents may come from teaching and research institutions in France or abroad, or from public or private research centers.
L'archive ouverte pluridisciplinaire HAL, est destinée au dépôt et à la diffusion de documents scientifiques de niveau recherche, publiés ou non, émanant des établissements d'enseignement et de recherche français ou étrangers, des laboratoires publics ou privés. 


\title{
A la recherche de la centralité perdue*
}

\section{In search of lost centrality}

\author{
Lise BOURDEAU-LEPAGE \\ Chargée de recherche, Dr HDR \\ Cemagref, DTM \\ 2, rue de la papeterie B.P.76 - 38402 Saint-Martin-d'Hères Cedex \\ lise.bourdeau-lepage@cemagref.fr \\ Jean-Marie HURIOT \\ Jacky PERREUR \\ Professeurs \\ Université de Bourgogne, Laboratoire d'Economie et de Gestion \\ B.P. 26611 - 21066 DIJON Cedex \\ huriot@u-bourgogne.fr / jacky.perreur@u-bourgogne.fr
}

Mots-clés : Centralité, villes, réseaux, globalisation

Keywords: centrality, cities, networks, globalization

Classification JEL : R00, R12

La pluralité des centres

Ordonnée aux rouages

Des multiples systèmes

Contrarie en nous

Un leurre

Fascination

D'un possible seul Centre

Pivot de tous les autres

(André Verdet, L'obscur et l'ouvert)

*Première version août 2007. 


\section{Résumé}

Les mutations majeures de l'organisation de l'espace économique semblent remettre en question le concept de centralité et ses applications tout particulièrement à l'échelle de la ville, des réseaux de villes et de l'économie globalisée. L'étalement urbain fait émerger une centralité diffuse, multiple. La globalisation recompose les flux matériels et immatériels, et redistribue le pouvoir économique au bénéfice des villes globales. Une analyse précise des termes «centre » et «centralité » et des phénomènes qu'ils recouvrent permet de montrer que les schémas classiques, convenablement adaptés, restent pertinents à l'échelle des villes, mais que de nouveaux schémas de centralité apparaissent bien au niveau mondial, essentiellement sur une base réticulaire.

\section{Summary}

The major changes of economic space organization seem to cast doubt over the concept of centrality and its applications, especially at the city, city network and global economy scales. Urban sprawl gives rise to a diffused or multiple centrality. Economic globalization reorganizes material and immaterial flows, and redistributes economic power to the advantage of global cities. A precise analysis of the terms "center" and "centrality" and of the phenomena they refer to permits to show that the classical patterns, if they are properly adapted, remain relevant at the urban scale, but that new centrality patterns are emerging at the world scale, on a reticular basis. 


\section{Introduction : La centralité en question}

La centralité, clé longtemps incontournable de l'ordonnancement spatial des sociétés, a été malmenée par les mutations majeures des espaces contemporains. La fascination pour la recherche du centre unique, à chaque échelle spatiale, semble perdre de sa puissance. La centralité deviendrait-elle un leurre ? Métropolisation, globalisation, nouvelles technologies de l'information, semblent s'être liguées pour anéantir les vieux schémas spatiaux, et lorsqu'on parle encore de centralités, elles apparaissent toujours «nouvelles », «multiples », « diffuses » ou « éclatées ».

L'étalement urbain ferait dépérir les centres des villes, pour créer des structures urbaines multicentriques de plus en plus complexes, pour faire apparaître des conurbations, des régions urbaines, où le centre tendrait à disparaître. La révolution de l'information a déstabilisé les observateurs au point que certains, à une époque, ont prédit le plus sérieusement du monde la fin des villes, la ville à la campagne, la liberté de localisation, l'éparpillement général. Les mutations de l'ère de l'information feraient du monde lui-même une structure multicentrique, voire a-centrique. Michel SERRE évoque la diffusion de l'urbain sur la totalité de la planète : « le globe tend à devenir une seule ville », qu'il appelle Villeneuve, «cité invisible, dont le centre est partout et la circonférence nulle part » (SERRE, 1993). A l'échelle du monde, depuis longtemps, l'Europe n'est plus centrale que sur les représentations déformées des planisphères. Aujourd'hui la triade Amérique du Nord - Europe - Japon est elle-même remise en question par l'émergence des Nouveaux Pays Industrialisés. Le centre du monde semble de plus en plus instable, de plus en plus diffus.

La centralité serait-elle moribonde ? Certainement pas, à condition qu'on la pense en se détachant à la fois des vieux schémas et des effets de mode. La fin des villes n'est pas pour demain, et la concentration urbaine ne fait que s'accentuer. Toutefois les formes urbaines ont changé. Elles sont partout multicentriques, dit-on. En fait les choses sont plus complexes, et la rhétorique du multicentrisme perd de son pouvoir de persuasion. Les structures monocentriques n'ont pas réellement disparu. Elles réapparaissent sous d'autres modalités. Le discours sur le multicentrisme avait seulement oublié le sens du mot «centre », ou l'avait vidé d'une partie de son contenu. Au niveau mondial, il faut repenser la centralité en termes de réseaux. La centralité n'est plus matérialisée en un lieu, ou en un ensemble de lieux, mais dans un système de relations entre des lieux spécifiques, les villes globales (BOURDEAULePage et Huriot, 2005b, SASSEN, 1996, TAYLOR, 2004).

Voila posée la question sémantique. Il faut bien s'attaquer aux définitions, et retourner à la très riche signification du centre (HURIOT et PERREUR, 1994a, 1994b, 1997). Il est banal de dire que la centralité est polysémique. On dit de même de tous les grands concepts de l'analyse spatiale et en particulier de la ville et de la région. La centralité, inévitablement liée à l'agglomération des hommes et des activités, partage avec celle-ci le statut de concept majeur de l'analyse spatiale. L'idée de centralité est présente dans presque toutes les disciplines, en sciences exactes comme en sciences humaines, mais elle est plus universelle que pluridisciplinaire. Dans nos disciplines, la centralité, le centre, sont les fondements de concepts plus élaborés comme le schéma centre-périphérie, les structures monocentriques, les centres secondaires, la hiérarchie des lieux centraux, etc. Il faut s'interroger sur la manière dont ces concepts sont en mesure de rendre compte des mutations spatiales contemporaines.

Dans ce papier nous rappelons d'abord la richesse sémantique du centre et de la centralité et l'efficacité analytique de ces concepts (1). Puis nous évoquons les grandes théories liées à 
la centralité : les analyses centre-périphérie, les modèles monocentriques et multicentriques et la théorie des lieux centraux (2). Enfin nous examinons dans quelle mesure ces modèles sont aptes à rendre compte des centralités d'aujourd'hui, à deux échelles spatiales : la ville et le monde (3). Nous pensons en effet qu'il s'agit aujourd'hui des deux échelles les plus pertinentes de l'analyse spatiale. Une défense de la primauté de l'échelle urbaine a été proposée dans BouRdEAU-LEPAGE et HuRIOT (2007). L'échelle mondiale va de soi dans le contexte de la globalisation.

\section{-1 - \\ La centralité polysémique}

La centralité est un concept universel, une clé de lecture des structures, des inégalités, des interactions asymétriques, dans nombre de disciplines allant de la littérature aux sciences exactes et aux mathématiques. Dans les seules sciences humaines, elle apparaît en sociologie, en droit, en histoire, en géographie, en économie. Limitons-nous à la centralité comme clé de lecture de l'organisation spatiale des hommes et des activités. Restons-en à la question conceptuelle, sans évoquer le passage aux mesures concrètes, qui sont largement présentées et discutées dans la littérature. L'idée de centre est une construction mentale, une fiction analytique qui traduit sous forme simple des formes spécifiques observables d'organisation spatiale mais qui présente aussi une haute valeur symbolique qu'on ne peut négliger, tant elle est liée à la dimension observable. Cette section présente la conception classique de la centralité, celle de la recherche d'un centre.

\subsection{Les dimensions observables}

Un centre peut être défini par plusieurs critères pris séparément ou dans différentes combinaisons (HURIOT et PERREUR 1994a, 1994b, 1997)

\section{L'accessibilité}

Le centre est d'abord un concept géométrique. L'idée de centre renvoie communément au centre du disque, ou au milieu d'un segment. Plus généralement, dans un ensemble discret de points (en particulier dans un graphe), ou dans un espace continu mais limité par des frontières, le centre est le point qui possède la meilleure position relative, en termes de distances aux autres points. C'est celui qui par exemple minimise la somme des distances à tous les autres points ou la plus grande distance à un des autres points. A titre anecdotique, c'est dans cet esprit qu'en France, le chef lieu de département a été en principe choisi de façon que tout citoyen ait la possibilité de rencontrer le représentant de l'Etat de son département en se déplaçant en une journée maximum (à cheval).

Notons qu'en l'absence de frontières, un espace continu n'a pas de centre : sur la surface de la sphère ou sur un cercle, tous les points ont la même position relative. Clairement, le centre défini en termes d'accessibilité est entièrement dépendant de l'espace de référence (notamment de l'échelle) et du type de distance utilisée. En tout cas, en sciences régionales, le seul critère de la distance, fût-elle exprimée en temps ou en coût, est pauvre et en général insuffisant pour identifier correctement un centre. 


\section{L'agglomération}

Le centre est un lieu de concentration maximum de population, d'emploi, d'activités économiques et/ou culturelles, de création de richesse (PIB par exemple), de connaissances, de capacités d'innovation, de l'information et de son traitement, du pouvoir de décider et de contrôler, dans l'ordre politique, juridique ou économique. Le centre est ainsi le lieu où est aggloméré l'élément le plus important pour l'analyse que l'on mène. Le centre diffère en principe selon l'élément choisi. En général, il ne se confond pas avec le centre d'accessibilité, mais compte tenu de l'interdépendance entre agglomération et réseaux de transport et communication, les lieux d'agglomération maximum sont souvent aussi les lieux d'accessibilité maximum. Concrètement, la plupart des critères d'agglomération aboutissent naturellement à la ville, où se concentre une grande variété d'individus et d'activités.

\section{L'accessibilité et l'agglomération}

Position et concentration peuvent être associées quand on cherche la meilleure accessibilité à des entités économiques inégalement concentrées dans un ensemble de lieux. De nombreux indicateurs combinent la distribution spatiale d'une variable et les distances entre les lieux. On raisonne alors en référence plus ou moins directe avec le principe gravitaire et avec les mesures de potentiels. Ces termes spécialisés ne recouvrent rien de plus que des mesures de position des lieux pondérées par leurs poids respectifs (voir annexe). Dans tous les cas, on peut déterminer une échelle de centralité où le centre correspond à une valeur extrême.

\section{Les interactions}

Finalement, les critères précédents cachent ce qui est certainement l'essentiel de la centralité pour l'économiste et le géographe. Un centre est un lieu privilégié d'interactions, de rencontres, de confrontations, d'échanges matériels et immatériels. L'agglomération crée la proximité entre les hommes et entre les activités, et favorise ainsi les interactions directes par le contact face-à-face, nécessaire à l'échange d'informations complexes et personnalisées, c'est-à-dire « tacites ».

L'accessibilité, par l'ensemble des réseaux de transport et de communication, conditionne les interactions entre le centre et les autres lieux. Le centre attire et/ou diffuse des flux humains, matériels ou immatériels. Il attire hommes et activités; il diffuse production, connaissances, informations et décisions. Les interactions induisent des infrastructures destinées à rendre le centre plus accessible, et favorisent l'agglomération. Ainsi le centre résulte des interactions et crée des interactions. On est dans la logique cumulative de formation et de croissance des villes. La ville est en effet la concrétisation la plus manifeste de cette centralité combinant accessibilité et agglomération pour maximiser les interactions internes aussi bien qu'externes. Nous y reviendrons. Soulignons seulement ici que la centralité d'interactions est difficile à mesurer directement. En particulier, les interactions immatérielles, qui aujourd'hui fondent la spécificité urbaine, sont pratiquement impossibles à saisir directement. Elles sont souvent approximées par des mesures d'agglomération et/ou de position, ou par des sources supposées d'interaction (TAYLOR, 2004).

\section{La domination}

Des précédents critères de centralité, il découle que le centre domine les autres lieux. Il est le lieu le plus accessible, il concentre au maximum tout ce qui est important, tout ce qui a de la valeur, tout ce qui est stratégique et prestigieux. Il maximise les interactions internes et externes. Il est un lieu de décision et de contrôle. L'idée de centralité véhicule donc nécessairement celle d'inégalité. Cela se manifeste en particulier dans les concepts dérivés 
centre-périphérie, de modèle monocentrique et de lieux centraux (section 2). Le centre entretient avec les autres lieux des interactions asymétriques.

\subsection{La part de l'imaginaire}

L'idée de centre a inévitablement une dimension symbolique, elle traduit non seulement une observation mais des symboles, des représentations mentales qui engendrent une rhétorique littéraire et cartographique de la centralité où réel et imaginaire se confondent étrangement.

\section{Symboles et représentations mentales}

Chaque lieu a une signification qui dépasse celle de sa pure description. Chaque lieu est source de représentations mentales qui incluent des jugements de valeur par rapport à des référents culturels au sens le plus large, faisant appel à l'esthétique, à l'histoire, au pouvoir, au prestige. Ces représentations ont une dimension symbolique. Un symbole est un objet spatial intégrant «une dimension signifiante au-delà d'une simple fonction organisatrice »; il y a espace symbolique dès qu'un lieu est chargé d'une signification «collectivement et volontairement élaborée »; (MONNET, 2000). MONNET définit sur ces bases une centralité symbolique basée sur l'importance symbolique des lieux ressentie par les individus.

Le symbole peut être dans l'objet spatial lui-même et/ou dans les fonctions qu'il assure. Un monument historique, esthétique, majestueux, sera symbole par lui-même et par ce qu'il a représenté dans le passé, même s'il n'abrite plus aucune fonction importante. Un objet symbolique peut valoriser un lieu précis (l'obélisque et la place de la Concorde) ou un espace plus vaste (la tour Eiffel, symbole de Paris). La centralité symbolique d'un lieu est d'autant plus grande que ce lieu abrite un plus grand nombre d'objets symboliques, des objets plus importants ou renvoyant à des valeurs plus élevées (MONNET, 2000). Mais la concentration d'activités économiques stratégiques ou d'activités culturelles de grande renommée est également créatrice de centralité symbolique. Indépendamment des bâtiments, Wall Street est le symbole de la finance globalisée. La destruction du World Trade Center à Manhattan a touché un des plus grands symboles de l'économie globale.

Lorsque fonctions symboliques et objets symboliques correspondent, la centralité n'en est que renforcée. Le prestige de l'objet symbolique suscite l'agglomération des classes aisées et/ou des fonctions stratégiques. La centralité symbolique se double alors d'une centralité sociale ou économique. Cette correspondance caractérise particulièrement les centres des villes européennes, et ce n'est peut-être pas étranger au fait que les fonctions supérieures sont plus présentes dans les centres des villes en Europe qu'ailleurs, qu'aux Etats-Unis par exemple (section 3).

Toutefois, «il est difficile, voire vain, d'établir si un espace est symbolique parce qu'il a une valeur centrale, ou s'il est central parce qu'il a une valeur symbolique » (MONNET, 2000, p. 413).

\section{La rhétorique de la centralité}

Que veut-on dire quand on qualifie un lieu de central ? Quel message veut-on faire passer, outre celui de la simple connaissance d'un ordre spatial existant? Au-delà de la description, il y a la symbolique. Le lieu élevé au rang de centre est valorisé. Du constat on passe au jugement de valeur, du plus au mieux. On voit bien quel sens peut prendre cette hiérarchie dans une société malade du classement et du record. Cette dimension symbolique, qui parle à 
l'imaginaire, appelle inévitablement la rhétorique. Le désir de persuader peut alors mettre en danger le sérieux scientifique de la recherche du centre. Le choix d'une approche du centre ou d'une méthode de détermination peut alors être justifié uniquement par ce qu'on veut dire, par la volonté de persuader qu'un lieu est central. Le problème est pris à l'envers : étant donné un lieu, comment montrer qu'il est central ? Il suffit de bien choisir l'espace de référence et la mesure de la centralité.

Avec le seul critère de la distance entre les capitales européennes, on peut placer Prague au centre de l'Europe (voir annexe). Il y a quelques années, le Conseil Général de Saône et Loire et le Conseil Economique et Social d'Île-de-France ont chacun publié une carte montrant que son territoire était au centre de l'Europe, alors que $300 \mathrm{~km}$ les séparent. Ainsi, des régions européennes, bien que différemment localisées, cherchent à améliorer leur attractivité en se forgeant une centralité illusoire basée sur une fausse évidence visuelle. Publicité mensongère ? Sans aller jusque là, on peut voir là l'inversion d'une logique qui voudrait que l'attractivité définisse la centralité et non que l'on suscite l'attraction en posant a priori un postulat (faux) de centralité. La puissance persuasive de ce genre de démarche tient à deux choses : d'abord à la valeur symbolique attribuée à l'idée même de centre : si on est au centre, on est le meilleur; elle tient ensuite à la simplicité du procédé : quoi de plus persuasif que de se placer au centre d'une série de cercles concentriques ? Etre au centre d'un espace limité par un cercle, c'est bien, peu importe comment on a tracé le cercle. Plus étonnant : sur internet (http://www.escapades.be/esca/mcu/mcuceur.htm), on peut découvrir la photo d'un curieux monument ainsi commentée «A quelques kilomètres de OIGNIES, sur la route de FUMAY, vous pourrez voir ce monument de verre réalisé par le sculpteur Bernard Tirtiaux. Il symbolise le centre exact (sic) de l'Europe des quinze. Malheureusement, dès une nouvelle adhésion à l'idéal communautaire, le dit centre se déplacera de quelques dizaines ou centaines de kilomètres. ». On se passe de commentaire...

Symbolique et rhétorique sont plus subtilement associées dans ce qui paraît pourtant la représentation du monde la plus sérieuse, le planisphère. Il faut d'abord souligner que le passage de la sphère terrestre au planisphère implique bien plus qu'une déformation des continents : il introduit des limites, celle du rectangle plan, alors que la sphère est un espace sans limites. Sans limites, une sphère ne possède pas de centre. Un planisphère possède un centre. Pratiquement tous les planisphères placent l'Europe au centre dans la partie supérieure de la carte, voire au centre de la carte, en trichant sur la position de l'équateur. "L'Europe, qui est à l'origine de la mondialité, a ordonné la figure de la terre autour d'elle»(GRATAlOUP, 2002, p. 843). C'est de la rhétorique cartographique ! Cette représentation n'est pas innocente. «La pensée que nous avons du monde est contrainte par les images qui nous le donnent à voir. En retour, cette image mentale contribue, parce qu'elle guide les acteurs, à la production de la centralité réelle »(GRATALOUP, 2002, p. 843). C'est en se fondant sur de telles représentations que les vieux manuels scolaires expliquaient que la France se trouvait «au centre des terres émergées». Plus loin de nous encore, Jérusalem figurait au centre du monde sur les fameuses cartes médiévales « $\mathrm{T}$ dans $\mathrm{l}$ 'O ». On a aussi produit des planisphères où le Sud est ... en haut. Notre vision du monde en est bouleversée. Et pourquoi pas un planisphère centré sur le Pôle Nord? Mais c'est une représentation encore plus clairement connotée, où le Nord apparaît central et le Sud périphérique ! Tout cela montre bien qu'une carte est «un révélateur des symboles et des pouvoirs » (BAILLY et SCARIATI, 1999, p. 42) ainsi qu'un révélateur de notre conception de la centralité. 


\subsection{La centralité multidimensionnelle}

Il ressort de cette section qu'un centre peut avoir une signification très partielle, s'il ne répond qu'à l'un des critères évoqués. Il y a alors une multitude de centralités, différant selon l'échelle spatiale et selon l'élément qui est privilégié. Dans ce sens, la Cité de Londres est un centre financier, Rotterdam un centre portuaire, Salzburg un centre musical, et Las Vegas le centre mondial du jeu et du mauvais goût.

Mais on peut aussi chercher un centre multifonctionnel, multidimensionnel. Les grandes villes des pays développés sont des centres dans la plupart des sens du terme. Elles agglomèrent une population variée, des activités spécialisées diversifiées y compris les services les plus avancés et les fonctions de décision et de contrôle. Elles abritent le pouvoir économique, mais aussi l'innovation, et les activités culturelles de niveau national ou international. Elles permettent toutes les formes d'interaction, quel que soit leur objet, quel que soit leur support. Elles sont des centres pour une gamme très large de critères reposant sur l'agglomération comme sur la position, les interactions et la valeur symbolique. Londres est ainsi un des trois principaux centres financiers du monde (SASSEN, 1996), mais aussi un des deux principaux centres européens de rayonnement international (ROZENBLAT et CiCILLE, 2003), le centre mondial du trafic aérien (AIRPORT COUNCIL INTERNATIONAL, 2005) et le centre mondial de la connectivité des services supérieurs (TAYLOR et al., 2002). Ainsi, au niveau supérieur, les centralités se rejoignent. Cependant, au niveau du système des villes globales, les villes dont les interactions externes ont une portée mondiale, la centralité ne peut être décrite uniquement en termes de la conception classique développée dans cette section. Ce sera l'objet de la section 3 de revenir sur cette idée. Auparavant, nous devons évoquer quelques modèles significatifs dérivés du concept premier de centre.

\section{$-2-$ \\ La centralité modélisée : variations sur le thème centre-périphérie}

L'idée de centre est explicitement présente dans quelques modèles fondamentaux de l'analyse spatiale, en géographie et en économie. La modélisation suppose une simplification, et les modèles de la centralité effacent le plus souvent une grande partie de la richesse sémantique du concept. Cette section n'examine que quelques exemples de modèles formels ou non, à titre illustratif. Elle ne prétend ni à l'exhaustivité ni à la généralité. Nous rappelons d'abord les principes des modèles centre-périphérie et de ses dérivés en économie géographique et en économie urbaine avec le modèle monocentrique, puis nous évoquons les modèles à centres multiples et hiérarchisés, parmi lesquels le modèle des lieux centraux.

\subsection{Centre et périphérie}

Le schéma spatial le plus simple basé sur l'idée de centre est l'opposition centrepériphérie. Dès qu'on détermine un centre unique, la périphérie en est son complémentaire naturel. Est périphérique tout espace qui n'est pas central. Le modèle centre-périphérie est une métaphore qui sert à mettre de l'ordre dans le chaos du monde (GuILLOREL, 2001). Elle traduit une ségrégation spatiale simplifiée à l'extrême, ou une relation asymétrique. Comme la plupart des schémas simples, celui-là a un fort contenu symbolique, et souvent une connotation idéologique. Le centre est positif : il est attractif, prestigieux, riche, développé, 
innovant, dominant, etc. ; la périphérie est le négatif de cette image (HuRIOT et PERREUR, 1997).

PREBISCH (1950) aurait inauguré l'analyse de type centre-périphérie en analysant les relations asymétriques entre d'une part l'Amérique Latine et plus généralement les économies spécialisées dans les produits primaires, à la périphérie du système économique mondial, et d'autre part les grands pays industriels, au centre du système. Le concept est repris par FRIEDMAN (1966) dans son étude sur le Venezuela. Son opposition centre-périphérie repose sur des critères techniques, institutionnels et culturels. Dès cette époque, il rejette l'idée reçue de la convergence et souligne les facteurs de freinage ou de blocage de la réduction des inégalités. "Les forces non contrôlées d'une économie de marché dynamique semblent s'opposer à une convergence du centre et de la périphérie »(FRIEDMAN, 1966, cité par GuILLOREL, 2001, p. 217). Cette analyse, plus ou moins adaptée, se retrouve dans une partie de l'économie du développement et dans l'analyse de la division internationale du travail.

La métaphore centre-périphérie est universelle. Elle permet d'interpréter l'histoire du monde grâce au concept d'économie-monde, comme elle représente une structure ordonnée produite par le processus d'agglomération dans les modèles de l'économie géographique. Ainsi BRAUDEL et KRUGMAN se rejoignent à ce niveau métaphorique.

\subsection{L'économie-monde}

BRAUDEL (1979) utilise un schéma centre-périphérie pour interpréter la structure spatiale de l'«économie-monde» du XVIe au XVIIIe siècle. L'économie-monde est un concept explicatif d'une réalité qui a toujours existé. L'économie-monde est «un fragment de l'univers, un morceau de la planète économiquement autonome, capable pour l'essentiel de se suffire à lui-même et auquel ses liaisons et ses échanges intérieurs confèrent une certaine unité organique.» (BRAUDEL, 1979, tome 3, p. 12). C'est un «univers en soi » dont l'autonomie rappelle celle de l'État Isolé de vON THÜNEN.

Cet espace est limité par des barrières naturelles ou par l'effet de «distances hostiles ». Il est organisé autour d'un centre qui est un pôle urbain, un centre d'affaires. Il existe des villesrelais autour du centre principal, « associées ou complices, plus fréquemment encore asservies à leur rôle second »(BRAUDEL, 1979, tome 3, p. 20). Le centre correspond toujours à un pouvoir fort.

«Toute économie-monde est un emboîtement, une juxtaposition de zones liées ensemble, mais à des niveaux différents. Sur le terrain, trois «aires », trois catégories au moins se dessinent: un centre étroit, des régions secondes assez développées, pour finir d'énormes marges extérieures. Et obligatoirement les qualités et caractéristiques de la société, de l'économie, de la technique, de la culture, de l'ordre politique changent selon qu'on se déplace d'une zone à l'autre »(BRAUDEL, 1979, tome 3, p. 28)

«Le centre, le "coeur", réunit tout ce qui existe de plus avancé et de plus diversifié. L'anneau suivant n'a qu' une partie de ces avantages, bien qu'il y participe : c'est la zone des "brillants seconds". L'immense périphérie, avec ses peuplements peu denses, c'est au contraire l'archaïsme, le retard, l'exploitation facile par autrui. Cette géographie discriminatoire, aujourd'hui encore, piège et explique l'histoire générale du monde, bien que celle-ci, à l'occasion, crée aussi d'elle-même le piège par sa connivence » (BRAUDEL, 1979, tome 3, p. 28). 
«Il y a bien sûr, des périphéries partout de par le monde »(BRAUDEL, 1979, tome 3, p. 29) «Cependant, les zones arriérées ne sont pas distribuées exclusivement dans les vraies périphéries. En fait, elles criblent les régions centrales elles-mêmes de multiples taches régionales » (BRAUDEL, 1979, tome 3, p. 30). Voilà un schéma qui reste très actuel à l'heure de la globalisation.

\subsection{Agglomération et centre-périphérie}

Passons de l'échelle mondiale à l'échelle régionale, et de l'interprétation historique à l'économie formalisée pour rencontrer le modèle de base de l'économie géographique, dit «modèle centre-périphérie ». A nouveau, voici un centre où s'agglomère ce qui fait le développement, l'activité industrielle, et une périphérie qui reste agricole. On n'est pas si loin de Prebisch et de Friedman. Le modèle de Krugman (1991) pose deux régions au départ identiques, sans épaisseur spatiale, donc assimilables à des points. Il s'agit donc de deux lieux au sens le plus abstrait.

L'agriculture est répartie à égalité dans les deux localisations. On cherche à savoir comment se localise l'activité industrielle, sachant que chacune des nombreuses firmes identiques est soumise à des rendements croissants et doit approvisionner la demande des deux lieux. Dans chaque région, la demande est issue des agriculteurs et des travailleurs industriels. Ainsi deux mécanismes vont jouer. (1) Chaque producteur réalise un arbitrage entre rendements croissants et coûts d'approvisionnement des marchés; les rendements incitent à concentrer l'industrie dans un unique lieu tandis que les coûts de transport favorisent la proximité aux marchés, donc la dispersion; (2) la demande dans un lieu est d'autant plus importante que de nombreux producteurs industriels y sont localisés, et inversement les producteurs recherchent une localisation où la demande est importante.

De (1) il découle qu'un coût de transport faible et des rendements croissants importants favorisent l'agglomération en un seul lieu ; (2) implique qu'une part importante de l'activité industrielle soutient également l'agglomération, et que le processus d'agglomération est cumulatif. L'égale répartition de l'industrie dans les deux lieux est un équilibre possible. Mais on montre que si, toutes choses égales d'ailleurs, on a un niveau suffisamment élevé des rendements croissants et/ou de la part de l'industrie, et/ou un niveau suffisamment bas des coûts de transport, un petit déséquilibre en faveur d'un des deux lieux met en route un processus cumulatif qui aboutit à la concentration de toute l'industrie dans un seul lieu, l'autre restant uniquement agricole.

Ce schéma en soi n'est pas nouveau, pas plus que le mécanisme cumulatif d'aggravation des inégalités, déjà décrit par HIRSHMAN (1958) et MYRDAL (1970). Ce qui l'est, c'est la modélisation du mécanisme de formation de la structure centre-périphérie. Dans les conditions précitées, un ordre centre-périphérie se forme de lui-même, suite à la moindre inégalité initiale. On se situe donc ici en amont de toutes les conceptions précédentes, en

mettant en lumière les facteurs favorables à ce mécanisme (pour plus de détails et de commentaires, FUJITA et THISSE, 2003). 


\subsection{La ville monocentrique}

Le modèle urbain monocentrique (AlONSO, 1964, FuJITA, 1989 ; PAPAGEORGIOU, 1990) est l'héritier du schéma des cercles de VON THÜNEN (HURIOT, 1994). Dans les deux cas, on se donne un centre : une ville-marché chez VON THÜNEN, un centre d'emploi pour la ville monocentrique. Dans les deux cas, en l'absence de perturbation directionnelle, l'espace s'ordonne régulièrement autour de ce centre unique: des cercles de culture pour vON THÜNEN, un gradient de prix du sol et de densité, voire des cercles de revenus dans la ville monocentrique. Dans l'un et l'autre modèles, le centre est le seul lieu dominant: il est le passage obligé et exclusif de toutes les interactions. La structure centre-périphérie est donc postulée dans une version extrême d'absolue dépendance de la périphérie par rapport au centre, dépendance qui nécessairement se retrouve au niveau des structures spatiales résultantes. Dans les deux approches, le centre est le marché, des produits agricoles dans l'une, de l'emploi dans l'autre. Le marché détermine tout le reste et sa localisation dans un centre unique postulé détermine une périphérie nécessairement radio-concentrique. Derrière l'analyse mathématique, la charge symbolique est puissante: le centre domine de façon absolue parce qu'il est le lieu du marché. Ce marché est concurrentiel, or on sait que le marché concurrentiel est un écran entre des agents qui n'ont aucune interaction directe. Le schéma centre périphérie est ici tel qu'il n'existe aucune relation possible dans l'espace périphérique, le centre polarisant la totalité des interactions. Ainsi le centre apporte sa propre symbolique et s'accapare une autre symbolique, celle du marché.

\subsection{La pluralité des centres}

De la ville monocentrique postulée, on passe à la ville multicentrique résultat d'un processus d'agglomération. Des centres multiples mais identiques on passe, sans égard pour la chronologie, à une hiérarchie de centres avec la théorie des places centrales puis avec l'endogénéisation de cette hiérarchie par FUJITA, KRUGMAN et MORI (1999).

\section{Multiplier les centres}

L'essentiel du changement n'est pas dans le nombre de centres mais dans le fait que les modèles montrent comment les interactions entre les agents, consommateurs et producteurs, produisent une centralité. Il ne s'agit pas ici de faire le panorama de ces modèles, issus des travaux de FuJita et OGAwA (principalement 1982 ; FuJita, 1994, FuJITA et THISSE, 2003) à la suite de ceux de BECKMANN (1976) et BORUKHOV et HOCHMAN (1977), mais seulement de les replacer dans la problématique de la centralité.

La centralité est maintenant endogène. Elle résulte du jeu combiné des avantages de l'agglomération des firmes en termes d'échange d'information et des coûts occasionnés par cette agglomération en termes de transport de la main-d'œuvre et de rente foncière.

La forme de centralité obtenue dépend essentiellement de la manière dont les coûts d'échange d'information entre les firmes et les coûts de déplacement des travailleurs varient avec la distance. L'agglomération centrale des firmes est d'autant plus marquée que les premiers sont élevés par rapport aux seconds. La décroissance des coûts de communication inter-firme est donc un facteur de déconcentration. Des configurations clairement multicentriques apparaissent seulement si les coûts de communication entre les firmes décroissent de façon amortie avec la distance (fonction exponentielle négative). 
Une telle approche est seulement en mesure de montrer la possibilité qu'il existe plusieurs centres, compte tenu de l'existence d'équilibres multiples pour des combinaisons données de coûts. Comme dans le modèle monocentrique, le centre est considéré comme une agglomération de firmes, toutes identiques. Des centres multiples sont donc également tous identiques. La centralité reste conçue sur la base d'une relation de firmes à travailleurs, ceuxci n'ayant aucune relation directe entre eux. Ils sont pourtant en concurrence pour l'occupation du sol. Mais là, chaque individu est face au marché, pas en interaction directe avec les autres individus. A peu de choses près, on a démultiplié le schéma centre-périphérie sans en changer la logique.

\section{Hiérarchiser les centres}

Dès le XVIIIe siècle, CANTILLON avait décrit une véritable hiérarchie fonctionnelle d'agglomérations à quatre niveaux : la ville capitale, la ville, le bourg, le village (CANTILLON, 1755, Huriot et PERreur, 1992). Les géographes ont systématisé l'idée avec la théorie des lieux centraux (Christaller, 1933; LÖSCH, 1940; BERRY, 1967 ; PuMAIN, 1992). L'économie spatiale formalisée a suivi, dans les années1990.

Un lieu central a pour fonction essentielle de desservir son arrière-pays. C'est une concentration d'activités de production, due aux rendements croissants, et un ensemble de relations extérieures : la centralité est celle de la ville. La théorie des lieux centraux explique l'existence d'une hiérarchie de villes sur la base d'une hiérarchie de fonctions urbaines. Différents niveaux de fonctions urbaines sont définis selon leur spécialisation, donc selon l'extension de leur aire de marché. Dans sa forme la plus simple, la hiérarchie obtenue est telle que tout centre d'un rang donné fournisse tous les biens de ce rang et des rangs inférieurs. L'organisation spatiale de cette hiérarchie donne lieu chez CHRISTALLER et chez LÖSCH (1940) à la structure hexagonale devenue curieusement symbole des Sciences Régionales de Langue Française.

Ce principe a donné lieu à une vaste littérature. Retenons seulement le passage à la théorie de la genèse des systèmes hiérarchiques de villes (FUJITA, KRUGMAN et MORI, 1999) dans une approche qui combine la microéconomie de la formation des villes et une certaine dose de théorie évolutionniste. La structure urbaine engendrée par le modèle reproduit celle de CHRISTALlER, aux hexagones près, puisqu'on se situe ici dans un espace linéaire et non dans les deux dimensions du plan.

Cette analyse hiérarchique reste elle aussi attachée à la conception classique de la centralité. A l'échelle spatiale de la plus grande aire de marché, il existe un et un seul centre de niveau supérieur et toute cette région dépend de lui. C'est une conception très «territoriale» de la centralité. On est toujours dans une variation sur le thème centrepériphérie, où différents niveaux de centralités s'emboîtent les uns dans les autres.

\subsection{La fascination du centre}

La recherche de la centralité est dominée par la fascination du centre unique. Le schéma centre-périphérie, même démultiplié et hiérarchisé, reste soumis à cette fascination. On a pu constater que cela oriente les modélisations aussi bien que les représentations mentales. Une telle situation suggère deux remarques, relatives à l'épistémologie et à l'utopie.

$\mathrm{Au}$ niveau épistémologique, cette fascination relève d'un biais plus général, qui est la volonté de trouver la cause première et unique de tout phénomène. Cela relève d'un 
essentialisme aujourd'hui dénoncé par une bonne partie de la philosophie des sciences. Il semble que la centralité, si elle est conçue comme la recherche $d u$ centre, ou comme une variante du schéma centre-périphérie, et quand elle est élevée au niveau de principe premier de l'explication de l'organisation de l'espace, relève de ce biais. Nous avons tous des difficultés à sortir de nos habitudes de pensée. On n'est pas loin de l'idée d' «obstacle épistémologique » à la formation de l'esprit scientifique de BACHELARD (1938).

Les utopies sociales, urbaines dans leur quasi-totalité, accordent une place de choix à l'organisation spatiale selon un schéma simple souvent inspiré du principe centrepériphérie (BAUMONT et HURIOT, 1997). La ville est circulaire et le pouvoir est localisé en son centre. L'idée d'ordre est à son zénith. Le dualisme centre-périphérie est la projection spatiale d'une structure sociale très hiérarchisée. C'est aussi le moyen de réaliser un ordre social hiérarchique. Le centre, symbole du principe premier, est le lieu qui domine tout. C'est le lieu du pouvoir car sa localisation maximise l'efficacité du contrôle. Cette structure spatiale est présente dans l'Atlantide de Platon, dans nombre de projets utopiques de la Renaissance aussi bien que dans la ville de Chaux dessinée au XVIIIe siècle par Ledoux, dont seul un demi cercle a été construit à la saline d'Arc-et-Senans, autour du bâtiment central du directeur.

\section{- 3 - \\ LA CENTRALITE OBSERVEE}

Comment les représentations de la centralité évoquées précédemment rendent-elles compte de l'organisation actuelle de l'espace et de ses mutations majeures ? Comme il a été suggéré plus haut, on peut considérer que l'évolution actuelle de l'organisation spatiale se situe principalement à l'échelle des agglomérations urbaines et à celle du monde globalisé. Les deux échelles sont d'ailleurs étroitement liées l'une à l'autre. Dans ce qui suit, l'analyse de la centralité est confrontée à ces deux échelles.

\subsection{Quelle centralité dans la ville ?}

Deux phénomènes caractérisent l'évolution des villes contemporaines : l'étalement et la multipolarisation. Toutes les villes s'étalent. Mais cet étalement n'est pas uniforme et un examen plus fin révèle la formation de concentrations de population et d'activités autour de la ville d'origine. La question est de savoir si une telle évolution peut s'analyser en termes de structures multicentriques. Il s'avère pour plusieurs raisons que le modèle «multicentrique » ne rend compte que partiellement des structures urbaines et certainement pas avec la même pertinence en différentes régions du monde. L'accent est mis ici sur les villes françaises et européennes.

\section{L'étalement urbain}

C'est une dispersion de la population et des activités économiques, donc une baisse apparente de la centralité. La population et l'emploi s'accroissent plus vite à la périphérie qu'au centre de la ville. Ils peuvent diminuer de façon absolue au centre. On peut observer ce phénomène partout dans le monde. Il est particulièrement visible dans les interminables banlieues des villes américaines, qui rassemblent la moitié de la population totale des Etats Unis. 
L'étalement urbain est illustré ici par le cas français (HURIOT, 2004 ; BOURDEAU-LEPAGE et HURIOT, 2006). Quelques constats suffisent à comprendre l'ampleur du phénomène. Dans toutes les villes, le gradient de densité baisse. L'extension spatiale des villes est plus que proportionnelle à l'augmentation de leur population, ce qui veut dire que leur densité moyenne diminue. La plus forte croissance de la population se situe en zone péri-urbaine, plus qu'en banlieue : de 1962 à 1999, la population de l'espace péri-urbain a été multipliée par 7. Cette croissance a été la plus forte dans les plus grandes villes. Toutefois, la période 19901999 est marquée par un léger retour au centre. L'emploi s'étale également, mais moins que la population. Entre 1978 et 1997, Paris a perdu 18\% de ses emplois ; dans le même temps, la petite couronne en gagnait 5\% et la grande couronne 40\%. Cependant, la densité d'emploi en 1997 est encore 110 fois plus élevée à Paris que dans la grande couronne.

On assiste ainsi à une dispersion des villes, jusque dans les lointaines zones péri-urbaines. Mais, malgré un très fort taux de croissance périphérique, population et emploi restent nettement plus denses au centre qu'en périphérie, lorsqu'on raisonne en moyenne en fonction de la distance au centre ou dans des couronnes concentriques. Les chiffres précédents sont biaisés par un effet taille. En chiffres absolus, l'emploi s'est le plus accru dans les communes les plus proches de Paris (GILLI, 2004). Mais cette approche concentrique est insuffisamment précise. La périphérie des villes n'est pas isotrope.

\section{La ville multipolaire}

Toutes les descriptions et analyses des espaces urbains mettent l'accent sur leur multipolarité, que ce soit au niveau d'une agglomération (aire urbaine par exemple) ou d'une région urbaine plus vaste. L'émergence de «nouvelles centralités » urbaines a fait couler de l'encre et suscité bien des discours. Mais il ne s'agit pas toujours de nouvelles «centralités ». Les esprits ont été profondément marqués par les révélations de GARREAU (1991) sur les fameuses edge cities, véritables nouvelles villes apparaissant spontanément autour des villes américaines selon un modèle voué à se reproduire partout dans le monde. L'attrait du nouveau et la fascination pour cette «multiplication des centres» ont produit des erreurs d'appréciation.

Il est vrai que de nouvelles concentrations d'emploi se sont rapidement développées dans les périphéries de toutes les villes du monde, particulièrement dans les plus grandes. Mais ces concentrations ne reproduisent que rarement les caractéristiques de la ville-centre initiale. Revenons à la définition d'un centre. Le centre est multidimensionnel, c'est un lieu de pouvoir, de domination, de prestige. Il abrite des activités économiques de rang supérieur, il est chargé de symboles. En Île-de-France, la concentration d'emplois de Roissy, celle de Chessy (Euro Disney) ou celle d'Aubergenville (Renault) ressemblent-elles à des centres dans ce sens plein ? De simples concentrations d'emplois ne forment pas nécessairement des centres. Alors appelons-les des pôles et réservons le terme de centre pour les pôles qui concentrent une variété suffisante d'activités, en particulier supérieures, de création de décision et de contrôle, et qui de ce fait exercent une domination économique. Alors une structure multipolaire n'est pas nécessairement multicentrique. L'examen attentif des villes françaises, sur la base des nombreuses études disponibles, montre que les pôles sont clairement différenciés entre eux, et différents du pôle principal, classiquement central. Le pôle de la ville-centre, même s'il perd des emplois, se spécialise dans les activités du plus haut niveau, clairement plus que les autres, et ceci d'autant plus qu'on se situe dans une grande ville. Le dépérissement des centres n'est pas à l'ordre du jour (CHAPAIN et POLESE, 2000). La division du travail entre les pôles est géographiquement organisée selon un schéma centre-périphérie. On aboutit à un schéma «multipolaire-monocentrique » (BOURDEAU- 
LEPAGE et HurIot, 2005a, et pour l' ̂̂le-de-France : HALBERT, 2007). Il s'agit effectivement d'une nouvelle organisation de l'espace urbain, mais pas vraiment d'une multicentralité.

D'une autre manière, l'étude POLYNET nuance le caractère multicentrique de huit grandes régions urbaines du Nord-Est européen, sur la base de leur degré d'intégration évalué par les diverses connexions entre les pôles urbains qui les composent (en particulier les interactions entre services avancés à la production). En général, ces connexions sont plus faibles qu'on aurait pu penser. Mais le degré de multicentrisme est très variable d'une région urbaine à l'autre. Il est relativement faible pour les régions primatiales comme le Bassin Parisien. Cette région ressemble plus à un système urbain monocentrique qu'à un système fonctionnellement intégré.

\section{Les vertus du multicentrisme en question}

Longtemps le multicentrisme a été prôné par les discours officiels, au nom de la déconcentration, du désengorgement des grandes villes. On aurait pu penser qu'une structure urbaine multipolaire, à population égale, permettrait de rapprocher la population de l'emploi, limitant ainsi les déplacements et l'encombrement des voies radiales, tout en préservant les économies d'échelle au niveau de chaque pôle. Le développement de la multipolarité en France ne s'est pas accompagné d'une diminution des déplacements, bien au contraire : de 1982 à 1999, le pourcentage de travailleurs qui se rendent à leur travail dans une autre commune ou un autre département a augmenté, ainsi que les distances moyennes parcourues (TALBOT, 2001).

D'autres vertus supposées sont mal corroborées. Au niveau national, il n'y a pas de liaison significative entre les performances économiques et le multicentrisme (VANDERMOTTEN, 2003). Au niveau des conurbations, pour une population donnée, les structures multicentriques sont moins bien dotées en services avancés et fonctions supérieures de commandement : "elles entretiennent des éparpillements qui empêchent d'accéder à des effets de seuils et aux effets multiplicateurs qui y sont associés » (VANDERMOTTEN, 2005).

Enfin, la dispersion multicentrique est indirectement remise en cause par la généralisation des plaidoyers sur la ville compacte et les avantages des fortes densités urbaines, en termes notamment de déplacements et d'environnement. A l'engouement pour la décentralisation succède la mode de la compacité et du retour aux fortes centralités, sans que l'argumentation soit nécessairement plus convaincante dans un cas que dans l'autre.

\subsection{Où est le centre du monde ?}

Limitons-nous à l'aspect économique de la question. Le contexte actuel est celui de la globalisation, ce qui signifie en particulier que les stratégies des firmes s'exercent au niveau du marché mondial et que les interactions sont réalisées en temps réel : un choc en un point du monde engendre des réactions immédiates partout ailleurs. Ainsi une économie globale est plus qu'une économie internationale ou mondiale. C'est «une économie capable de fonctionner comme unité en temps réel à l'échelle planétaire »(CASTELLS, 1998).

On a défendu ailleurs que ni la nation ni la région ne sont aujourd'hui les échelles d'analyse les plus pertinentes (BOURDEAU-LEPAGE et HuRIOT, 2007). Les échanges, matériels et surtout immatériels, se font plus entre les villes qu'entre les nations ou les régions. Les villes concentrent les fonctions de haut niveau d'innovation, de décision et de contrôle. Un petit nombre de villes exercent ces fonctions au niveau le plus élevé et avec le plus grand 
rayonnement : ce sont les «villes globales », c'est-à-dire les villes où les acteurs sont en mesure de coordonner des activités économiques complexes à l'échelle globale. Les villes globales ont ainsi le quasi-monopole d'un pouvoir économique qui s'appuie essentiellement sur les sièges sociaux de grandes firmes et les services supérieurs à la production. C'est « là où se joue l'économie mondiale, là où s'élaborent les stratégies globales, où se focalisent les interactions planétaires » (BOURDEAU-LEPAGE et HURIOT, 2005b).

Les villes globales ont deux caractéristiques majeures : elles concentrent les instruments du pouvoir économique, et elles sont fortement interconnectées. C'est sur la base de ces deux critères que sont réalisés les divers classements des villes globales, notamment par le groupe de recherche Globalization and World Cities (GaWC; TAYLOR, 2004). Ces classements donnent des hiérarchies qui pourraient faire penser que les villes globales forment une structure comparable à celle des lieux centraux de CHRISTALlER. Par exemple, le critère de la concentration des services supérieurs place les principales villes globales dans les catégories alpha-, béta- et gamma-world cities. Le critère de connectivité des services supérieurs donne un classement différent mais tout de même très lié au précédent, où Londres et New York sont de loin les villes les mieux connectées au reste de l'économie globale. Les villes globales sont donc des centres de pouvoir et d'activités supérieures. On est cependant assez loin de structures à la CHRISTALLER. Dans la hiérarchie Christallerienne, les villes de plus haut niveau restent très liées à leur arrière-pays. Ce n'est pas le cas des villes globales. Chaque ville globale, au niveau de ses activités supérieures, est en général plus fortement connectée aux autres villes globales qu'à son arrière-pays. L'organisation des villes globales répond à une logique complètement différente de celle des lieux centraux (DERUDDER et WiTLOX, 2004). Pour cette raison, TAYLOR (2001) remplace le terme de hinterland par celui de urban hinterworlds, qui représente le réseau des villes globales avec lesquelles une ville déterminée est connectée avec plus ou moins d'intensité. On imagine la complexité du réseau global obtenu en combinant les hinterworlds des 123 villes retenues dans l'analyse...

Toujours dans la logique hiérarchique, les villes de niveau supérieur regroupent à peu près les mêmes fonctions. Dans le système des villes globales, des spécialisations et complémentarités apparaissent. Le réseau global est différencié géographiquement. Les trois villes globales dominantes de SASSEN (1996; New York, Londres, Tokyo) ont chacune leur première place dans un domaine financier. Le secteur banque-finance est sur-représenté en Asie Pacifique et le conseil en gestion est très concentré aux Etats-Unis. L'examen de l'intensité des interactions permet d'identifier des sous-réseaux régionaux à forte cohésion interne, comme celui de l'Asie Pacifique ou de l'Europe de l'Est (TAYLOR, 2003). Le sousréseau européen est très diversifié et très ouvert sur le reste du monde. (TAYLOR et DERUdDER, 2003). L'Afrique, le Moyen-Orient et l'Asie non Pacifique restent à l'écart des principales connexions.

Dans ces conditions, que devient la centralité économique à l'échelle mondiale ? On peut difficilement la définir en termes d'un pays ou d'un groupe de pays. On ne peut non plus l'assimiler à une hiérarchie urbaine à la CHRISTALLER. Chaque ville globale n'a pas pour fonction première d'approvisionner son arrière-pays, et le système des villes globales ellesmêmes ne constitue pas non plus une hiérarchie christallerienne. Qu'est donc devenu le centre du monde ? Pour comprendre cette nouvelle (vraiment nouvelle) centralité, il faut abandonner le schéma hiérarchique qui a tant marqué les esprits, de même que le modèle centre-périphérie avec toute sa fascination. Ce qui fascine aujourd'hui, c'est une centralité de réseau. Ce n'est pas un lieu qui domine, c'est un réseau, ou un ensemble de sous-réseaux. Ce qui définit le centre, ce n'est plus une agglomération d'activités en un point de l'espace, c'est un système 
d'interactions soutenant le fonctionnement de l'économie globale. Alors le centre est-il nulle part ? Non, il est partout, du moins partout où les fonctions économiques supérieures sont connectées au reste du monde. En d'autres termes la centralité n'est plus d'essence territoriale, mais de nature réticulaire, interactive. La périphérie regroupe alors tous les espaces non (ou mal) connectés à ce réseau-centre, c'est-à-dire les villes et les pays qui restent à l'écart de la globalisation.

Le centre du monde, tel qu'il vient d'être défini, fait penser au «noyau » d'un graphe, dans le sens où toute ville périphérique est dominée par au moins une ville du noyau et qu'il n'y a pas de domination marquée entre les villes du noyau.

\section{Conclusion}

La centralité classique est encore un instrument d'analyse pertinent, en particulier au niveau d'une agglomération urbaine ou d'une conurbation. A cette échelle, le vieux schéma monocentrique n'est pas entièrement dépassé. Les structures dites multicentriques cachent souvent des espaces multipolaires-monocentriques. Les centres des villes ne se vident pas, ils se re-spécialisent dans les activités de niveau supérieur. Cette centralité observée rejoint la centralité symbolique : «toutes les villes sont concentriques; mais aussi, conformément au mouvement même de la métaphysique occidentale, pour laquelle tout centre est le lieu de la vérité, le centre de nos villes est toujours plein : lieu marqué, c'est en lui que se rassemblent et se condensent les valeurs de la civilisation : la spiritualité (avec les églises), le pouvoir (avec les bureaux), l'argent (avec les banques), la marchandise (avec les grands magasins), la parole (avec les agoras : cafés et promenades) »(BARTHES, 1970, p. 43 ; cité par MONNET, 2000, p. 409).

La centralité doit être plus profondément repensée au niveau mondial. « On ne peut donc pas penser la centralité dans le Monde comme dans l'espace d'une ville ou même d'un pays. L'idée d'un "centre Ville ", même multinodal, ou d'une capitale du Monde ne s'impose guère, d'abord parce que les liens entre les territoires urbains de cet «archipel » ne sont pas eux-mêmes territoriaux. » (Dolfus et al., 2002, pp. 863-64). De la centralité classique, territoriale, et de son schéma associé centre-périphérie, il faut passer à une centralité de réseau. L'idée de réseaux de villes globales est déjà bien ancrée dans les esprits, mais elle est encore peu opérationnelle. L'analyse quantitative de ces réseaux bute encore sur une difficulté majeure : celle de la mesure des interactions, plus ou moins contournée par les travaux du groupe GaWC. Quant aux symboles, on les trouvera peut-être dans l'idéal d'ubiquité... partagé seulement par ceux, bien peu nombreux, qui sont les maîtres de la globalisation.

\section{Bibliographie}

AIRPORT COUNCIL INTERNATIONAL, 2005, Worldwide airports ranking, 2005 Preliminary Report.

Alonso W., 1964, Location and Land Use, Harvard University Press, Cambridge.

BACHELARD G., 1938, La formation de l'esprit scientifique. 12e édition (1983), Vrin, Paris.

Bailly A., ScARIATI R., 1999, Voyage en Géographie, Anthropos, Paris.

BAumont C., Huriot J.-M., 1997, "La Ville, la Raison et le Rêve : entre théorie et utopie", L'espace Géographique, 2, pp.99-117.

BeCKMAnN M.J., 1976, Spatial Equilibrium in a Dispersed City, in Papageorgiou Y.Y. ed., Mathematical Land Use Theory, Lexington Books, Toronto, pp. 117-125.

BERRY B.J.L., 1971, Géographie des marchés et du commerce de détail, A. Colin, Coll. U, Paris. Traduit de Geography of Market Centers and Retail Distribution, 1967, Prentice-Hall, Englewood Cliffs. 
Borukhov E., Hochman O., 1977, "Optimum and Market Equilibrium in a Model of a City Without a Predetermined Center”, Environment and Planning, A, vol. 9, nº, pp.849-856.

BouRdEAU-LEPAGE L., 2007, "Advanced services and city globalization on the Eastern fringe of Europe", Belgeo, $\mathrm{n}^{\circ}$ 1, pp.133-146.

Bourdeau-Lepage L., Huriot J.-M., 2005a, "On poles and Centers: cities in French Style", Urban Public Economics Review, n³, pp. 13-36.

BourdeAu-LePAGE L., HuRiot J.-M., 2005b, La métropolisation, thème et variations, in Buisson M.-D., Mignot D. eds, Concentration économique et ségrégation spatiale, De Boeck Université, Bruxelles, pp. 39-65.

CANTILlON R., 1755, Essai sur la nature du commerce en général, Fletcher Gyles, Londres. Réimpression, 1892, Macmillan, Londres.

CASTElls M., 1998, La société en réseaux, Fayard, Paris. Traduit de The Rise of the Network Society, Blackwell, Oxford, 1996.

Chapain C., POlese M., 2000, "Le déclin des centres-villes : mythe ou réalité ? Analyse comparative des régions métropolitaines nord-américaines”, Cahiers de Géographie du Québec, vol. 44, n¹23, pp. 303-324.

CHRISTAller W., 1933, Die Zentralen Orte in Suddeutschland, Iéna, Traduction anglaise par Baskin C.W., 1966, Central Places in Southern Germany, Prentice-Hall, Englewood Cliffs.

DERUDDER B., WITLOX F., 2004, "Assessing central places in a global age: on the networked localization strategies of advanced producer services”, Journal of Retailing and Consumer Services, vol. 11, n³, pp. 171180.

Dolfus O., Grataloup C., LEVy J., 2002, Un monde de nouvelles centralités, in TIDE-CNRS, Le monde et la centralité, Actes 2, gravure sur CD, 851-868. En ligne: http://halshs.archivesouvertes.fr/docs/00/15/00/03/PDF/Le_Monde_et_la_Centralite\%283\%29.pdf

FRIEDMANN J., 1966, Regional development policy: a case study of Venezuela, Cambridge (Mass.).

FuJiTA M., 1989, Urban Economic Theory - Land Use and City Size, Cambridge University Press, Cambridge.

FuJITA M., 1994, L’équilibre spatial - L’interaction entreprises ménages, in Auray J.-P., Bailly A., Derycke P.H., Huriot J.-M., sous la direction de, Encyclopédie d'économie spatiale, Economica, Paris, pp. 213-223.

FujtTa M., Krugman P., Mori T., 1999, "On the Evolution of Hierarchical Urban Systems", European Economic Review, vol. 43, n², pp. 209-251.

FuJiTA M., ThISSE J.-F., 2003, Economie des villes et de la localisation, de Boeck, Bruxelles. Traduit de Economics of Agglomeration. Cities, Industrial Location and Regional Growth, 2002, Cambridge University Press, Cambridge.

Fujita M., OGAWA H., 1982, "Multiple Equilibria and Structural Transition of Non-Monocentric Urban Configurations", Regional Science and Urban Economics, vol. 12, n², pp. 161-196.

GARREAU J., 1991, Edge City: Life on the New Frontier, Anchor Books, Doubleday, New York.

GILli F., 2004, Choix de localisation des entreprises et périurbanisation des emplois, Thèse de Doctorat d'économie, Université de Sciences et Technologies, Lille.

GRATALOUP C., 2002, L'héritage encombrant de l'ancienne centralité européenne, in TIDE-CNRS, Le monde et la centralité, Actes 2, gravure sur CD, 841-850. En ligne: http://halshs.archivesouvertes.fr/docs/00/15/00/03/PDF/Le_Monde_et_la_Centralite\%283\%29.pdf

GuILlOREL H., 2002, L'actualité des modèles centre-périphérie, in TIDE-CNRS, Le monde et la centralité, Actes 2, gravure sur CD, 211-222. En ligne: http://halshs.archivesouvertes.fr/docs/00/15/00/03/PDF/Le_Monde_et_la_Centralite\%283\%29.pdf

HALBERT L., 2007, "From sectors to functions: producer services, metropolisation and agglomeration forces in the Ile-de-France region", Belgeo, n 1 , pp. 73-93.

HIRSCHMANN A., 1958, The Strategy of Economic Development, Yale University Press, New Haven.

HURIOT J.-M., 2004, Concentration and dispersal of employment in French Cities, in Richardson H. W., Bae C.H.C., Urban Sprawl in Western Europe and United States, Ashgate, Aldershot, Burlington, pp. 159-184.

HURIOT J.-M., BouRdEAU-LEPAGE L., 2007, L'illusion régionale, communication au colloque de l'ASRDLF, Grenoble.

Huriot J.-M., PERreur J., 1992, "Richard Cantillon and the intuitive understanding of space", Sistemi Urbani, $\mathrm{n}^{\circ} 1-2-3$, pp. 61-75.

Huriot J.-M., Perreur J., 1994a, La centralité, in Auray J.-P., Bailly A., Derycke P.-H., Huriot J.-M., eds, Encyclopédie d'économie spatiale, Economica, Paris, pp. 47-53.

Huriot J.-M., Perreur J., 1994b, L’accessibilité, in Auray J.-P., Bailly A., Derycke P.-H., Huriot J.-M., eds, Encyclopédie d'économie spatiale, Economica, Paris, pp. 55-59.

Huriot J.-M., PERreur J., (1997), Centre et périphéries : fondements et critères, in Célimène F., Lacour C. eds, L'intégration régionale des espaces, Economica, Paris, pp. 63-84.

KRUGMAN P., 1991, "Increasing Returns and Economic Geography", Journal of Political Economy, vol. 99, n॰31, pp. 483-499. 
Monnet J., 2000, "Les dimensions symboliques de la centralité”, Cahiers de Géographie du Québec, vol.44, $\mathrm{n}^{\circ} 123$, pp. 399-418.

Myrdal G., 1970, The Challenge of World Poverty: A World Anti-Poverty Program in Outline, Vintage Books, New York.

PAPAgeORgiou Y., 1990, The Isolated City State. An Economic Geography of Urban Spatial Structure, Routledge, London et New York.

PREBISCH R., 1950, The economic development of Latin America and its principal problems, United Nations, Department of Economic Affairs, Economic Commission for Latin America, New York.

Pumain D., 1992, Les systèmes de villes, in Bailly A., Ferras R., Pumain D., eds, Encyclopédie de géographie, Economica, Paris, pp.645-663.

Rozenblat C., Cicille P., 2003, Les villes européennes. Analyse comparative, La documentation française, Paris.

SASSEn S., 1996, La ville globale. New York. Londres. Tokyo, Descartes, Paris. Traduit de The Global City, Princeton University Press, Princeton, 1991.

Tацвот J., 2001, "Les déplacements domicile-travail. De plus en plus d'actifs travaillent loin de chez eux", INSEE Première, $\mathrm{n}^{\circ} 767$.

TAYLOR P.J., 2000, "World cities and territorial states under conditions of contemporary globalization", Political Geography, vol. 19, n¹, pp. 5-32.

TAYLOR P.J., 2001, "Urban hinterworlds: geographies of corporate service provision under conditions of contemporary globalization", Geography, vol. 86, n 1, pp. 51-60.

TAYLOR P.J., 2003, Regionality in the World City Network, GaWC Research Bulletin 116 (Z), Loughborough University: Global and World Cities Study Group and Network. En ligne: http://www.lboro.ac.uk/gawc/rb/rb116.html

TAYLOR P.J., 2004, World City Network. A Global Urban Analysis, Routledge, London et New York.

TAYlor P.J., CATAlano G., WalKeR D.R.F., 2002, "Measurement of the World City Network", Urban Studies, vol. $39, \mathrm{n}^{\circ} 13$, pp. 2367-2376.

TAYLOR P.J., DERUDDER B., 2003, Porous Europe: European Cities in Global Urban Arenas, GaWC Research Bulletin 125 (A). Loughborough University: Global and World Cities Study Group and Network. En ligne : http://www.lboro.ac.uk/gawc/rb/rb125.html

VANDERMOTTEN C., 2003, Le polycentrisme dans une perspective historique, in Allain R., Baudelle G., Guy C., eds, Le polycentrisme, un projet pour l'Europe, Presses universitaires de Rennes.

VANDERMOTTEN C., 2005, Quelle mesure de la dimension internationale des villes européennes? Communication au colloque ASRDLF, Dijon.

\section{- Annexe - Centre et potentiel}

Selon la théorie de la gravitation appliquée à l'espace des flux, les interactions entre deux lieux $i$ et $j$ sont d'autant plus importantes que les poids (pouvoir attractif) de $i$ et de $j$ ( $X_{i}$ et $X_{j}$ ) sont importants et que la distance $d_{i j}$ entre $i$ et $j$ est faible. Une méthode possible de détermination d'un centre utilise le potentiel d'interactions d'un lieu $i$, fonction de toutes les interactions que ce lieu peut avoir avec les autres lieux. Le potentiel d'interaction est donné par:

$$
P I_{i}=X_{i} \sum_{j \neq i} \frac{X_{j}}{d_{i j}}
$$

Le maximum sur $i$ définit un centre.

Alors que la seule utilisation des distances euclidiennes donne Prague comme centre du réseau des capitales européennes (Europe des 27), le potentiel d'interaction (avec $X_{i}$ mesurant la population de la ville $i$ ) donne Londres, très excentré en termes des seules distances, alors que Prague a un potentiel d'interaction très faible (BOURDEAU-LEPAGE, 2007). 